\title{
A multicenter, randomized, double-blind, placebo- controlled phase ii trial of intravenous inflammasome inhibitor (NuSepin) for the treatment of COVID-19 patients
}

\section{Seung-Yong Seong ( $\sim$ seongsy@snu.ac.kr)}

Seoul National University College of Medicine https://orcid.org/0000-0002-3671-1029

Seunghwa Lee

Shaperon Inc. https://orcid.org/0000-0001-9991-5005

\section{Research Article}

Keywords: COVID-19, Pneumonia, inflammasome, NEWS2

Posted Date: November 16th, 2021

DOI: https://doi.org/10.21203/rs.3.rs-1014277/v1

License: (c) (1) This work is licensed under a Creative Commons Attribution 4.0 International License.

Read Full License 


\section{Abstract \\ Purpose}

Here we evaluated whether a novel inflammasome inhibitor targeting GPCR19 (NuSepin) provides greater benefit than placebo in patients with COVID-19 pneumonia.

\section{Methods}

We conducted a double-blind, randomized, placebo-controlled phase ii trial of intravenous NuSepin, which is an NLRP3 inflammasome inhibitor targeting GPCR19, in adults who were hospitalized with COVID-19 pneumoniae in Romania from September 2020 to March 2021. The primary outcome was the time to clinical improvement, defined as a decline of two ordinal scales (OS) from randomization on a sixcategory OS that ranges from 1 (discharged with normal activity) to 6 (death) (=TTCI_P). In addition, clinical improvement was also assessed by aggregated National Early Warning Score 2 (NEWS2), and TTCI_S was defined by time to NEWS2 $=0$ from randomization, which is maintained for $24 \mathrm{~h}$.

\section{Results}

TTCI_P was different between groups numerically, but not statistically. The median difference in TTCI_S was $3.5 \mathrm{~d}$ between the NuSepin group and the placebo group $(p=0.016)$ in moderate-to-severe patients (with baseline NEWS2 $\geq 5$ ) of the PP set [recovery rate ratio $=2.7, p=0.02$ ], which favored improved recovery in the NuSepin group. The overall recovery rate ratio was 3.4, which favors the NuSepin group in terms of recovery rate $(p=0.0026)$ when the effects of covariates (use of anti-viral drugs and baseline NEWS2 $\geq 5$ ) were adjusted. Serious adverse events were reported in a patient who received $0.2 \mathrm{mg} / \mathrm{kg}$ NuSepin (4.5\%), but this was found to be unrelated to NuSepin treatment.

\section{Conclusion}

Considering that NuSepin has a favorable and tolerable safety profile, clinical improvement of hospitalized moderate-to-severe patients might be achieved with $0.2 \mathrm{mg} / \mathrm{kg}$ NuSepin significantly faster than placebo.

\section{Take-home Message}

This is a first report of double-blind, randomized clinical trial in patients with COVID-19 to report the effects of inflammasome inhibitor targeting GPCR19 (NuSepin) compared with placebo. We found that NuSepin completely stabilize vital signs of moderate-to-severe COVID-19 patients without further supplemental oxygen therapy faster than placebo. 


\section{Introduction}

COVID-19 is caused by infection with severe acute respiratory syndrome-coronavirus 2 (SARS-CoV-2) [1]. More than 233 million cases and 4.8 million deaths have been confirmed globally in the last two years [2]. In severe cases of COVID-19 pneumonia, patients' situation is complicated by acute respiratory distress syndrome (ARDS), sepsis, septic shock, and multiorgan failures [3]. In the early phase of infection, treatment with anti-viral drugs are necessary to inhibit viral replication in the lung $[4,5]$. In comparison, anti-inflammatory drugs are needed to ameliorate lung inflammation caused by tissue destruction in delayed phases [6]. Therefore, anti-inflammatory drugs combined with anti-viral drugs are mandatory to decrease mortality and to expedite clinical recovery for a faster return to normal life without any sequalae [7].

To suppress systemic inflammation, WHO panels have recommended the use of systemic corticosteroids in patients with severe COVID-19 [8]. However, the WHO and the U.S. Food and Drug Administration (FDA) have warned that treatment of systemic corticosteroids could increase the risk of infection in COVID-19 patients [9]. Secondary infections, hyperglycemia, mood disorders, and avascular necrosis are also frequently found in COVID-19 patients who received dexamethasone [10,11]. Meta-analyses showed that steroids delayed viral clearance and increased long-term mortality by prothrombotic side effects in COVID-19 patients $[12,13]$. For these reasons, better anti-inflammatory drugs that have less adverse effects are urgently needed to manage COVID-19 patients.

Several anti-inflammatory drugs have been tested in COVID-19 patients [14]. Although Tocilizumab, i.e., anti-CD6 monoclonal antibody, decreased levels of IFN-y, IL-6 and TNF-a, its efficacy is controversial [1519]. Several Janus-associated kinase (JAK) inhibitors, anakinra and anti-cytokine antibody cocktails, are still under evaluation or have already been tested without successful outcomes [20,21].

We demonstrated that a GPCR19 agonist could attenuate systemic inflammation [22]. The GPCR19 pathways regulate inflammation by inhibiting inflammasomal activation[23]. Taurodeoxycholate (TDCA) is an active pharmaceutical ingredient of NuSepin and a GPCR19 agonist [24] that suppresses NLRP3 inflammasome activation [25]. NuSepin could alleviate systemic inflammation in a cecal ligation and puncture-induced or LPS-induced mice sepsis model [22]. In addition, NuSepin increases the number of myeloid-derived suppressor cells (MDSC) which suppress inflammation further [26]. These findings suggest that NuSepin resolves systemic inflammation due to sepsis [22].

In a phase i clinical trial, among the 39 subjects who received NuSepin®, there were no serious adverse events. All adverse events by NuSepin treatment resolved spontaneously without any treatment. In this study, we report an interim analysis of the safety and efficacy of NuSepin for the treatment of COVID-19 patients in randomized, double-blind, placebo-controlled phase ii trials.

\section{Methods}

\section{Trial design}


The efficacy and safety of two doses of NuSepin $(0.1 \mathrm{mg} / \mathrm{kg}$ and $0.2 \mathrm{mg} / \mathrm{kg})$ for the treatment of adult patients diagnosed with SARS-CoV-2 were evaluated. Enrollment began in September 2020 and ended in March 2021. Patients were recruited at five study centers across Romania. NuSepin was infused intravenously b.i.d. from day 1 through day 14 or until hospital discharge. All patients received standardof-care of the trial site. The protocol was approved by the Central Ethics Committee and Local Ethics Committee (LEC) at each site where applicable, the National Agency for Medicines and Medical Devices of Romania. The patients received anti-viral drugs solely according to primary investigators' discretion, included in the standards-of-care. Written informed consent was obtained from each patient or from the patient's legally authorized representative if the patient was unable to provide consent.

For the study protocol, the IXRS (IRT system) was used to randomize and assign subjects to one of the three respective treatment groups via a central randomization methodology.

\section{Participants}

Inclusion criteria: age between 18-80 years, SARS-CoV-2 infection confirmed by PCR for the first time within $144 \mathrm{~h}$ prior to randomization, pneumonia based on chest X-ray/CT scan, blood leukocyte count > $4.0 \times 10^{9} / \mathrm{L}$ and lymphocyte count $>0.7 \times 10^{9} / \mathrm{L}, \mathrm{SpO}_{2} \leq 94 \%$ on room air or $\mathrm{PaO}_{2} / \mathrm{FIO}_{2}$ ratio $<300$ $\mathrm{mmHg}$, and for females with a negative pregnancy test prior to treatment.

Exclusion criteria: alanine aminotransferase (ALT)/ aspartate aminotransferase (AST) $>5 \times$ ULN (upper limits of normal), reduced renal function with estimated glomerular filtration rate $<30 \mathrm{~mL} / \mathrm{min}$, pregnant or breast-feeding women, evidence of multiorgan failure, and steroid treatment for any reason within $72 \mathrm{~h}$ prior to enrolment. Patients who needed steroid treatment were withdrawn by exclusion criteria.

\section{Procedures}

From day -5 to 0 , the patients were screened to assess eligibility for inclusion and exclusion, and enrolled into any of the three treatment arms at day 1 . The test drugs were given until complete remission up to 14 d. After the treatment was discontinued, the patients were followed-up for another $15 \mathrm{~d}$ until the end of the study visit (EoS). The safety of test drugs was monitored by laboratory tests according to the discretion of the primary investigators. The routine laboratory tests were performed at a central laboratory of each trial site.

\section{Outcomes}

The primary outcome is time to clinical improvement (TTCl), defined as the time (in days) from randomization until a decline of two categories on a six-category ordinal scale (OS) of clinical status (TTCI_P). The six-category OS is as follows: (6) death; (5) ICU, requiring ECMO and/or invasive mechanical ventilation (IMV); (4) ICU/hospitalization, requiring non-invasive mechanical ventilation (NIV)/ high-flow nasal cannula (HFNC) therapy; (3) hospitalization, requiring supplemental oxygen (such as low-flow nasal cannula (LFNC) or facial mask); (2) hospitalization, not requiring supplemental oxygen; 
and (1) complete clinical remission, i.e., no fever, normal respiratory rate, oxygen saturation return to normal, and cessation of cough.

One of the secondary outcomes were time to clinical remission evaluated by NEWS2. TTCI_S is defined as the time (in days) from randomization until a day when the NEWS2 score reaches zero which is maintained for $24 \mathrm{~h}$, that represent normal consciousness and complete normalization of vital signs without supplementary oxygen. All serious adverse events were recorded from day 1 to EoS. Safety assessments were based on the incidence of treatment emergent adverse events (TEAEs) in all three treatment groups.

\section{Statistical analysis}

The safety analysis set (SAS) and the intention-to-treatment (ITT) set were defined for statistical analysis in the clinical study protocol approved by the EMA (ClinicalTrials.gov no.: NCT04565379). The SAS and the ITT set consists of all randomized patients $(\mathrm{N}=64)$. Because the baseline OS was two in three cases, the patients were excluded in the modified ITT ( $\mathrm{mITT})$ population $(\mathrm{N}=61)$. The per-protocol population (PP) included patients without any major protocol deviations described in the synopsis. Three patients in the $0.1 \mathrm{mg} / \mathrm{kg}$ NuSepin group and one patient in the $0.2 \mathrm{mg} / \mathrm{kg}$ NuSepin group, did not complete the study due to treatment with prohibited steroid. One patient was not included in the PP set since the primary endpoints could not be assessed. Two patients in the placebo group discontinued due to steroid treatment and a patient in the $0.1 \mathrm{mg} / \mathrm{kg}$ NuSepin group withdrew consent. They were not included in the PP set $(\mathrm{N}=54)$.

Median TTCls were analyzed with Kaplan-Meyer analysis, and statistical significance in differences between groups was tested using a log-rank test. Mean and standard error were analyzed without the censored data. The hazard ratio and statistical significance from the Cox proportional hazard regression model were used to estimate the recovery rate ratio (RR) between groups. CRP and cytokine levels were summarized by descriptive statistics, including the mean and standard error, and statistical significance was assessed using a Wilcoxon Rank sum test.

For the analysis of patients who received anti-viral drugs, data from patients who received remdesivir were not included because the number of patients was limited (one or two in each group). Two patients who received more than two types of anti-viral drugs were not included clarify the effects of a specific type of anti-viral drugs.

\section{Results}

\section{Patients}

The median age of the patients was 62 (0.1 mg/kg NuSepin), 59 (0.2 mg/kg NuSepin), and 63 (placebo) years-old (Fig. 1, Supplementary table 1). 45\% (0.1 mg/kg NuSepin), 55\% (0.2 mg/kg NuSepin), and 50\% 
(placebo) were male. $100 \%$ of patients were Caucasian. The patients had comorbidities at enrollment, most commonly hypertension [ $\mathrm{n}=35(55 \%)]$, obesity [n=19 (30\%)], and diabetes mellitus [ $\mathrm{n}=16(25 \%)]$.

17 patients $(27 \%)$ were mild (with baseline NEWS2 < 5), 11 patients $(17 \%)$ were moderate (with baseline NEWS2 $=5 \sim 6$ ), and 36 patients (56\%) were severe (with baseline NEWS2 $\geq 7$ ) at randomization day (Supplementary Table 2). No patients were with baseline OS $=5$, and one patient in each group had a baseline OS $=2$ (Supplementary Table 3). 38 patients (59.4\%) received anti-viral drugs, such as LopinavirRitonavir, Remdesvir, Favipiravir, etc. (Supplementary Table 4). 16 patients received immune modulators, such as tocilizumab, hydroxychloroquine, or anakinra, respectively (Supplementary Table 5).

\section{Primary outcome}

There were no significant differences in TTCI_P between groups. In this report, data for the efficacy of 0.1 $\mathrm{mg} / \mathrm{kg}$ NuSepin were not included because it did not show statistically significant differences with placebo in outcomes tested. In the mITT population, none of the patients recovered until day 4 when assessed by TTCI_P. At day $9,38.1 \%$ and $15.8 \%$ of patients recovered in the NuSepin group and the placebo group, respectively. The RR was 1.34 (95\% C.I., $0.7 \sim 2.6, p>0.05$ ), which favored improved recovery in the NuSepin group numerically. In the PP population, $42.1 \%$ and $17.6 \%$ of patients recovered at day 9 in the NuSepin group and the placebo group, respectively. The RR was 1.9 (95\% C.I., $1.0 \sim 3.9, p>$ $0.05)$, which favored improved recovery in the NuSepin group numerically. When moderate-to-severe patients of the PP population were analyzed, the mean \% decrease of OS at day 9 in the patients of the NuSepin group $(41.7 \pm 3.7)$ was significantly higher than the placebo group $(24.3 \pm 5.9, p=0.028$, Supplementary Fig. 1).

\section{Secondary outcome}

First, the effects of covariates on TTCI_S were evaluated by Cox proportional hazard regression analysis (Fig. 2). The baseline NEWS2 and use of anti-viral drugs significantly affected TTCI_S. In the patient stratum with baseline NEWS2 $\geq 5, \mathrm{RR}=2.7$ (95\% Cl: 1.2-6.4, $p=0.02)$. In the patient stratum who received anti-viral drugs, $\mathrm{RR}=3.7(95 \% \mathrm{Cl}: 1.4-10.2, p=0.01)$. When the significant covariates (baseline NEWS2 $\geq 5$ and use of anti-viral drugs) were adjusted, overall RR between NuSepin and placebo was 3.4 $(1.5-7.4, p=0.0026)$, which significantly favors the recovery rate of the NuSepin group in the PP population.

We analyzed covariate effects of baseline NEWS2 (Supplementary Fig. 2, Supplementary Table 6). In the ITT population, clinical remission of patients with baseline NEWS $2 \geq 5$ was significantly delayed compared to patients with baseline NEWS $2<5$. For this reason, we analyzed TTCI_S after stratifying the ITT population with baseline NEWS2.

When PP population was analyzed, mean \% decrease of NEWS2 at day 9 in the moderate-to-severe patients of the NuSepin group (89.4 \pm 3.0$)$ was significantly higher than the placebo group $(64.3 \pm 10.2, p$ $=0.041$, Supplementary Fig. 3). 
When clinical recovery of moderate-to-severe patients in the ITT population was analyzed, the median TTCI_S was $9.0 \mathrm{~d}$ [95\% C.I. 7.0 12.0] in the NuSepin group and $12.5 \mathrm{~d}$ [95\% C.I. 8.0 25.0] in the placebo group ( $p=0.086$ ) (Fig. 3A). The median TTCI_S was $9.0 \mathrm{~d}$ [95\% C.I. 7.0 11.0] in the NuSepin group and $12.5 \mathrm{~d}$ [95\% C.I. 8.0 25.0] in the placebo group $(p=0.016)$, when moderate-to-severe patients of the PP population were analyzed (Fig. 3B). In this subgroup of patients, RR was 2.7 (95\% C.I., $1.2 \sim 6.4, p=0.02$ ), which favors improved recovery in the NuSepin group significantly faster than the placebo group.

We next analyzed covariate effects of anti-viral drugs. No significant differences of TTCI_S between the groups were observed in patients of the ITT population who did not receive anti-viral drugs (Supplementary Table 7). When NuSepin was combined with anti-viral drugs (Lopinavir-Ritonavir or Favipiravir), median TTCl_S was $9.0 \mathrm{~d}$ [7.0 12.0], which is comparable with the group of placebo + antiviral drugs of $13.0 \mathrm{~d}[4.0 \sim \mathrm{ND}] \mathrm{d}(p=0.013)$, when the ITT population was analyzed (Fig. 3C). The RR in this subgroup was 3.5 [1.2 10.0], which favors the group of NuSepin + anti-viral drugs compared with the group of placebo + anti-viral drugs $(p=0.02)$. When NuSepin was combined with anti-viral drugs, median TTCI_S was $9.5 \mathrm{~d}$ [7.0 12.0], which is comparable to the group of placebo + anti-viral drugs 13.0 [10.0 ND] ( $p=0.007)$, when moderate-to-severe patients in the ITT population were analyzed (Fig. 3D). The RR in this subgroup was 4.2 [1.3 13.6], which favors the group of NuSepin + anti-viral drugs compared with the group of placebo + anti-viral drugs $(p=0.01)$.

When NuSepin was combined with Lopinavir-Ritonavir, median TTCI_S was $8.0 \mathrm{~d}$ [5.0 11.0], which is comparable to the placebo + Lopinavir-Ritonavir group of $12.5 \mathrm{~d}$ [4.0 ND] $(p=0.08)$, when the patients in the ITT population were analyzed (Supplementary Fig. 4A). The RR in this subgroup was 7.7 [1.1 54.0], which significantly favors the group of NuSepin + Lopinavir-Ritonavir compared with the group of placebo + Lopinavir-Ritonavir $(p=0.04)$. When NuSepin was combined with Favipiravir, median TTCI_S was $10.0 \mathrm{~d}$ [7.0 13.0], which is comparable with the placebo + Favipiravir group of $19.0 \mathrm{~d}$ [10.0 ND] ( $p=$ 0.07), when the patients in the ITT population were analyzed (Supplementary Fig. 4B). The RR in this subgroup was 3.8 [0.78 18.83], which favors the group of NuSepin + Favipiravir compared with the group of placebo + Favipiravir, numerically $(p=0.10)$.

We hypothesized that anti-viral drugs might be given more frequently to more severe patients than to less severe patients. Therefore, we analyzed the baseline NEWS 2 of patients and compared given prognoses of these patients. As expected, baseline NEWS2 was significantly higher in patients who were given antiviral drugs (Supplementary Fig. 5). The mean baseline NEWS2 of patients who received anti-viral drugs was $7.6 \pm 0.71$ (not given with NuSepin) and $8.4 \pm 0.62$ (given with NuSepin together). In comparison, the mean baseline NEWS2 of patients who did not receive anti-viral drugs was $4.5 \pm 0.75$ (not given with NuSepin) and $5.2 \pm 0.95$ (given with NuSepin together) (Supplementary Fig. 5A). These findings indicate that anti-viral drugs were given more often to more severe patients. Moreover, clinical remission of patients who received anti-viral drugs without NuSepin was delayed compared to patients who did not receive anti-viral drugs nor NuSepin (Supplementary Fig. 5B), suggesting that anti-viral drugs were given to patients with higher baseline NEWS2 whose clinical improvement was significantly delayed compared to patients who did not receive anti-viral drugs. 
Next, we compared supplemental oxygen-free days between the groups (Supplementary Fig. 6). When patients in the PP population were analyzed after adjusting the effects of baseline NEWS2, the RR was 2.2 (95\% C.I., 1.0 4.6, $p=0.04$ ), which favors longer supplementary oxygen-free days in the NuSepin group than in the placebo group.

CRP levels decreased after randomization both in the NuSepin group and the placebo group (Fig. 4). However, the CRP level in the NuSepin group was normalized faster than that in the placebo group. At day 4 , CRP levels in $52 \%$ of patients in the NuSepin group returned within normal range, which is comparable to $20 \%$ of patients in the placebo group. When patients who received anti-viral drugs were analyzed, CRP levels in $64.3 \%$ of patients in the NuSepin group returned within the normal range at day 4 , which is comparable to the $11.1 \%$ of the placebo group (Supplementary Fig. 7 ).

At EoS, blood pro-inflammatory cytokines levels were significantly returned within normal range in the NuSepin group, and the percentage of patients with pro-inflammatory cytokines within normal ranges was higher in patients in the NuSepin group compared with those in the placebo group (Fig. 4). For example, IL-8 levels in patients of the NuSepin group (12.8 \pm 2.2$)$ at EoS were significantly lower than those of the randomization day $(22.1 \pm 4.0, p<0.05)$. In comparison, IL-8 levels in patients of the placebo group were not significantly decreased at EoS day $(259.4 \pm 167.8)$ compared with those of randomization day (22.0 \pm 3.2$)$. IL-8 levels returned within normal ranges in $55 \%$ of patients in the NuSepin group and in $33 \%$ of patients in the placebo group. At EoS day, the levels of TNF-a in patients of the NuSepin group were (7.4 \pm 0.7$)$ significantly lower than those of patients in the placebo group $(24.8 \pm 12.3, p=0.007)$, and TNF- a levels returned within normal ranges in $35 \%$ of patients in the NuSepin group and in $11 \%$ of patients in the placebo group. Furthermore, IL-6 levels returned within normal ranges in $60 \%$ of patients in the NuSepin group and in $33 \%$ of patients in the placebo group. IL- 6 levels in patients in the NuSepin group at EoS $(9.7 \pm 5.1)$ were significantly lower than those of randomization day $(19.0 \pm 3.9, p<0.05)$. Because no deceased cases were observed in either the NuSepin or placebo groups, we were unable to compare 28-d mortality.

\section{Safety Outcomes}

In the SAS population, which includes all 64 patients, $25 \%$ of patients experienced more than one adverse event (AE) (Table 1). The total number of events was $31.27 .3 \%$ (six patients, 12 events) of the $0.1 \mathrm{mg} / \mathrm{kg}$ NuSepin group, $27.3 \%$ (six patients, 12 events) of the $0.2 \mathrm{mg} / \mathrm{kg}$ NuSepin group, and $20.0 \%$ (four patients, seven events) of the placebo group reported at least one treatment-emergent AE. AEs were mild and recovered by EoS. The relationship between $13 \%$ AEs and $0.2 \mathrm{mg} / \mathrm{kg}$ NuSepin was determined to be 'possible' when graded as "probable, possible, or definite". There was one serious AE of dyspnea in the 0.2 $\mathrm{mg} / \mathrm{kg}$ NuSepin arm which was "not related" to NuSepin. The most common AE was increases in ALT, i.e., one case (5\%), two cases (9\%), and two cases (10\%) in the $0.1 \mathrm{mg} / \mathrm{kg}$ NuSepin, $0.2 \mathrm{mg} / \mathrm{kg}$ NuSepin, and placebo arm, respectively.

\section{Discussion}


After adjusting significant baseline imbalances of covariates (use of anti-viral drugs and baseline NEWS2), the overall recovery rate ratio (RR) was 3.4 -fold higher in the $0.2 \mathrm{mg} / \mathrm{kg}$ NuSepin group, compared with the placebo group, suggesting a higher probability of clinical improvement by NuSepin. Interestingly, the RR between the NuSepin group and the placebo group was $7.7(p=0.04)$ when the patients who received Lopinavir-ritonavir were analyzed after adjusting covariate effects of age $(\geq 60$ years-old). The median difference in TTCI_S was $9 \mathrm{~d}(p=0.07)$ between the NuSepin group and the placebo group when patients who received Favipiravir in the ITT set were analyzed. In the NuSepin group of the ITT population, there were no significant differences in median TTCI_S between patients who received anti-viral drugs and patients who did not $(p=0.58)$. Considering these findings in aggregate, the synergistic effects of NuSepin and anti-viral drugs is not conclusive with current data. Indeed, when combined with anti-viral drugs, the increase in statistical power and in the differences of TTCI_S between the NuSepin group and the placebo group might be attributable to selection of patients with higher baseline NEWS2.

The results of RCTs vary depending on definitions of disease severity, clinical remission, primary outcomes, inclusion and exclusion criteria, and type of standard care. Mortality might be an outcome that is less influenced by individual RCT than any other outcome. The recent drastic reduction in mortality may possibly be due to vaccination $[27,28]$. This suggests that a higher number of patients need to be enrolled to compare mortality between test therapeutics and placebo. Considering that novel therapeutics for COVID-19 should reduce mortality, number of severe/critical cases and hospital/ICU stays, a surrogate composite outcome measure that can represent improvement of clinical status of patients is required to expedite clinical development [29].

The quick Sequential Organ Failure Assessment (qSOFA), NEWS2, CURB-65, and systemic inflammatory response syndrome (SIRS) criteria are commonly used to evaluate clinical status of COVID-19 patients [30]. Six vital signs (i.e., respiratory rate, oxygen saturation, systolic blood pressure, pulse rate, level of consciousness (or new confusion), and body temperature), in addition to dependency on supplementary oxygen, are assessed by NEWS2 aggregate scoring. An advantage of NEWS2 compared to the other clinical tools is that both hypoxemia and supportive oxygen treatment are included as scoring parameters. For these reasons, NEWS2 has been recommended to be used for testing efficacy of novel therapeutics by the NHS [31]. NEWS2 showed better negative predictive value of $98.0 \%$ for early mortality [32] and superior accuracy to predict severity of COVID-19 than CURB-65, qSOFA, and COVID-GRAM score [33]. Taken together, rapid stabilization of vital sign with NuSepin might reduce days of hospital or ICU stays and mortality eventually.

\section{Conclusion}

Although it is difficult to compare efficacy directly between RCTs due to endpoints, inclusion criteria, etc., the efficacy of NuSepin for moderate-to-severe disease stratum appears to be superior to that of dexamethasone. In the RECOVERY trial, 1/RR (by 28-d mortality) between dexamethasone and placebo was 1.22 in the disease stratum receiving supplementary oxygen (OS $\geq 3$ in the NuSepin study) $[9,34-$ 
36], which is comparable to RR= 1.54 (by TTCI_S) in the mITT set and RR=1.96 (by TTCl_S) in the PP set of the NuSepin study (OS>=3). Considering that NuSepin was not associated with an increased risk of adverse events, NuSepin could be considered as relevant in the context of a pandemic, in which NuSepin modestly increases the day of stable vital signs after admission, and may reduce the risk of ventilatory complications, ICU length of stay, and burden on health care systems [37].

\section{Declarations}

\section{Author details}

${ }^{1}$ Department of Microbiology and Immunology, Seoul National University College of Medicine, Seoul, South Korea. ${ }^{2}$ Sf. Parascheva Infectious Diseases Clinical Hospital lasi Infectious Diseases Department, Iaşi, Romania. ${ }^{3}$ Sf. Ioan cel Nou" Suceava County Emergency Hospital, Infectious Diseases Department, Suceava, Romania, Stefan cel Mare University, Suceava, Romania. ${ }^{4}$ Ramnicu Sarat Clinical Hospital, Ramnicu Sarat, Romania. ${ }^{5}$ Leon Daniello Clinical Hospital for Infectious Diseases and Pneumophtisiology Victor Babes, Craiova Infectious Diseases Clinic for Adults, Cluj-Napoca, Romania. ${ }^{6}$ Shaperon Inc., Seoul, South Korea.

\section{Authors contributions}

Conception and design: SYS and HAS. Data collection: CL, CM, OACS and AC. Analysis and interpretation: SYS and SHL. All the authors edited and approved the manuscript.

\section{Funding}

This trial was supported by Shaperon Inc.

\section{Conflicts of interest}

CL, CM, OACS and AC: Grant from Shaperon Inc. for the submitted work. SHL and HSA are employees of Shaperon Inc. The patent "Composition for treating COVID-19 comprising taurodeoxycholic acid or pharmaceutically acceptable salts thereof as an active ingredient: PCT/KR2021/011236" was invented by SYS et.al. and applied by Seoul National University R\&DB Foundation. The exclusive license for the patent was transferred from SNU R\&DB Foundation to Shaperon Inc. SYS is a founder of Shaperon Inc. and its current CEO.

\section{Acknowledgements}

The first draft of the manuscript was derived from clinical science report written by Mariann.borsos of AdWare com and Dongjin Yoo by Symyoo.

\section{References}


1. WHO (2021) COVID-19 clinical management: living guidance. World Health Organization, Geneva

2. WHO (2021) Coronavirus (COVID-19) Dashboard. In: Editor (ed)^(eds) Book Coronavirus (COVID-19) Dashboard.World Health Organization, City, pp

3. Mokhtari T, Hassani F, Ghaffari N, Ebrahimi B, Yarahmadi A, Hassanzadeh G (2020) COVID-19 and multiorgan failure: A narrative review on potential mechanisms. J Mol Histol 51:613-628

4. Monari C, Gentile V, Camaioni C, Marino G, Coppola N, Vanvitelli C-G (2020) A Focus on the Nowadays Potential Antiviral Strategies in Early Phase of Coronavirus Disease 2019 (Covid-19): A Narrative Review. Life (Basel, Switzerland) 10

5. Taylor PC, Adams AC, Hufford MM, de la Torre I, Winthrop K, Gottlieb RL (2021) Neutralizing monoclonal antibodies for treatment of COVID-19. Nat Rev Immunol 21:382-393

6. Zhang W, Zhao Y, Zhang F, Wang Q, Li T, Liu Z, Wang J, Qin Y, Zhang X, Yan X, Zeng X, Zhang S (2020) The use of anti-inflammatory drugs in the treatment of people with severe coronavirus disease 2019 (COVID-19): The Perspectives of clinical immunologists from China. Clinical immunology (Orlando, Fla) 214:108393

7. Stebbing J, Phelan A, Griffin I, Tucker C, Oechsle O, Smith D, Richardson P (2020) COVID-19: combining antiviral and anti-inflammatory treatments. Lancet Infect Dis 20:400-402

8. WHO (2021) Corticosteroids for COVID-19. World Health Organization, Geneva

9. NIH (2021) Coronavirus Disease 2019 (COVID-19) Treatment Guidelines. U.S. National Institutes of Health, Bethesda

10. Villar J, Añón JM, Ferrando C, Aguilar G, Muñoz T, Ferreres J, Ambrós A, Aldecoa C, Suárez-Sipmann F, Thorpe KE, Jüni P, Slutsky AS (2020) Efficacy of dexamethasone treatment for patients with the acute respiratory distress syndrome caused by COVID-19: study protocol for a randomized controlled superiority trial. Trials $21: 717$

11. Suntronwong N, Thongpan I, Chuchaona W, Budi Lestari F, Vichaiwattana P, Yorsaeng R, Pasittungkul S, Kitphati R, Vongpunsawad S, Poovorawan Y (2020) Impact of COVID-19 public health interventions on influenza incidence in Thailand. Pathogens and global health 114:225-227

12. van Paassen J, Vos JS, Hoekstra EM, Neumann KMI, Boot PC, Arbous SM (2020) Corticosteroid use in COVID-19 patients: a systematic review and meta-analysis on clinical outcomes. Crit Care (London, England) 24:696

13. Mishra GP, Mulani J (2021) Corticosteroids for COVID-19: the search for an optimum duration of therapy. The Lancet Respiratory medicine 9:e8

14. Babaei F, Mirzababaei M, Nassiri-Asl M, Hosseinzadeh H (2021) Review of registered clinical trials for the treatment of COVID-19. Drug Dev Res 82:474-493

15. Danlos FX, Ackermann F, Rohmer J, Roumier M, Marabelle A, Michot JM (2021) High levels of TNFa in patients with COVID-19 refractory to tocilizumab. European journal of cancer (Oxford, England: 1990) 149: 102-104 
16. Price CC, Altice FL, Shyr Y, Koff A, Pischel L, Goshua G, Azar MM, McManus D, Chen SC, Gleeson SE, Britto CJ, Azmy V, Kaman K, Gaston DC, Davis M, Burrello T, Harris Z, Villanueva MS, Aoun-Barakat L, Kang I, Seropian S, Chupp G, Bucala R, Kaminski N, Lee Al, LoRusso PM, Topal JE, Dela Cruz C, Malinis M (2020) Tocilizumab Treatment for Cytokine Release Syndrome in Hospitalized Patients With Coronavirus Disease 2019: Survival and Clinical Outcomes. Chest 158:1397-1408

17. Rosas IO, Bräu N, Waters M, Go RC, Hunter BD, Bhagani S, Skiest D, Aziz MS, Cooper N, Douglas IS, Savic S, Youngstein T, Del Sorbo L, Cubillo Gracian A, De La Zerda DJ, Ustianowski A, Bao M, Dimonaco S, Graham E, Matharu B, Spotswood H, Tsai L, Malhotra A (2021) Tocilizumab in Hospitalized Patients with Severe Covid-19 Pneumonia. N Engl J Med 384:1503-1516

18. Zarinsefat A, Hartoularos G, Rychkov D, Rashmi P, Chandran S, Vincenti F, Yee CJ, Sarwal MM (2020) Single-Cell RNA Sequencing of Tocilizumab-Treated Peripheral Blood Mononuclear Cells as an in vitro Model of Inflammation. Front Genet 11:610682

19. Okoh AK, Bishburg E, Grinberg S, Nagarakanti S (2021) Tocilizumab use in COVID-19-associated pneumonia. J Med Virol 93:1023-1028

20. Jorgensen SCJ, Tse CLY, Burry L, Dresser LD (2020) Baricitinib: A Review of Pharmacology, Safety, and Emerging Clinical Experience in COVID-19. Pharmacotherapy 40:843-856

21. Gozzetti A, Capochiani E, Bocchia M (2020) The Janus kinase 1/2 inhibitor ruxolitinib in COVID-19. Leukemia 34:2815-2816

22. Chang S, Kim YH, Kim YJ, Kim YW, Moon S, Lee YY, Jung JS, Kim Y, Jung HE, Kim TJ, Cheong TC, Moon HJ, Cho JA, Kim HR, Han D, Na Y, Seok SH, Cho NH, Lee HC, Nam EH, Cho H, Choi M, Minato N, Seong SY (2018) Taurodeoxycholate Increases the Number of Myeloid-Derived Suppressor Cells That Ameliorate Sepsis in Mice. Frontiers in immunology 9: 1984

23. Kang JH, Kim M, Yim M (2021) FXR/TGR5 mediates inflammasome activation and host resistance to bacterial infection. Biochemistry and biophysics reports 27:101051

24. Tough IR, Schwartz TW, Cox HM (2020) Synthetic G protein-coupled bile acid receptor agonists and bile acids act via basolateral receptors in ileal and colonic mucosa. Neurogastroenterology and motility: the official journal of the European Gastrointestinal Motility Society 32:e13943

25. Yu SX, Chen W, Hu XZ, Feng SY, Li KY, Qi S, Lei QQ, Hu GQ, Li N, Zhou FH, Ma CY, Du CT, Yang YJ (2017) Liver $X$ receptors agonists suppress NLRP3 inflammasome activation. Cytokine 91:30-37

26. Krishnamoorthy M, Gerhardt L, Maleki Vareki S (2021) Immunosuppressive Effects of MyeloidDerived Suppressor Cells in Cancer and Immunotherapy. Cells 10

27. Christie A, Henley SJ, Mattocks L, Fernando R, Lansky A, Ahmad FB, Adjemian J, Anderson RN, Binder AM, Carey K, Dee DL, Dias T, Duck WM, Gaughan DM, Lyons BC, McNaghten AD, Park MM, Reses H, Rodgers L, Van Santen K, Walker D, Beach MJ (2021) Decreases in COVID-19 Cases, Emergency Department Visits, Hospital Admissions, and Deaths Among Older Adults Following the Introduction of COVID-19 Vaccine - United States, September 6, 2020-May 1, 2021. MMWR Morbidity and mortality weekly report 70: 858-864 
28. Scobie HM, Johnson AG, Suthar AB, Severson R, Alden NB, Balter S, Bertolino D, Blythe D, Brady S, Cadwell B, Cheng I, Davidson S, Delgadillo J, Devinney K, Duchin J, Duwell M, Fisher R, Fleischauer A, Grant A, Griffin J, Haddix M, Hand J, Hanson M, Hawkins E, Herlihy RK, Hicks L, Holtzman C, Hoskins M, Hyun J, Kaur R, Kay M, Kidrowski H, Kim C, Komatsu K, Kugeler K, Lewis M, Lyons BC, Lyons S, Lynfield R, McCaffrey K, McMullen C, Milroy L, Meyer S, Nolen L, Patel MR, Pogosjans S, Reese HE, Saupe A, Sell J, Sokol T, Sosin D, Stanislawski E, Stevens K, Vest H, White K, Wilson E, MacNeil A, Ritchey MD, Silk BJ (2021) Monitoring Incidence of COVID-19 Cases, Hospitalizations, and Deaths, by Vaccination Status - 13 U.S. Jurisdictions, April 4-July 17, 2021. MMWR Morbidity and mortality weekly report 70: 1284-1290

29. Altschul DJ, Unda SR, Benton J, de la Garza Ramos R, Cezayirli P, Mehler M, Eskandar EN (2020) A novel severity score to predict inpatient mortality in COVID-19 patients. Sci Rep 10:16726

30. Sperrin M, Grant SW, Peek N (2020) Prediction models for diagnosis and prognosis in Covid-19. BMJ (Clinical research ed) 369:m1464

31. Physicians, RCo (2017) National Early Warning Score (NEWS) 2: Standardising the assessment of acute-illness severity in the NHS. Updated report of a working party

32. Bradley P, Frost F, Tharmaratnam K, Wootton DG (2020) Utility of established prognostic scores in COVID-19 hospital admissions: multicentre prospective evaluation of CURB-65, NEWS2 and qSOFA. BMJ open respiratory research 7

33. Myrstad M, Ihle-Hansen H, Tveita AA, Andersen EL, Nygård S, Tveit A, Berge T (2020) National Early Warning Score 2 (NEWS2) on admission predicts severe disease and in-hospital mortality from Covid-19 - a prospective cohort study. Scand J Trauma Resusc Emerg Med 28:66

34. Bhimraj A, Morgan RL, Shumaker AH, Lavergne V, Baden L, Cheng VC, Edwards KM, Gandhi R, Muller WJ, O'Horo JC, Shoham S, Murad MH, Mustafa RA, Sultan S, Falck-Ytter Y (2020) Infectious

Diseases Society of America Guidelines on the Treatment and Management of Patients with COVID19. an official publication of the Infectious Diseases Society of America, Clinical infectious diseases

35. Lamontagne F, Agoritsas T, Siemieniuk R, Rochwerg B, Bartoszko J, Askie L, Macdonald H, Amin W, Bausch FJ, Burhan E, Cecconi M, Chanda D, Dat VQ, Du B, Geduld H, Gee P, Nerina H, Hashimi M, Hunt BJ, Kabra S, Kanda S, Kawano-Dourado L, Kim YJ, Kissoon N, Kwizera A, Leo YS, Mahaka I, Manai H, Mino G, Nsutebu E, Pshenichnaya N, Qadir N, Ranganathan SS, Sabzwari S, Sarin R, Sharland M, Shen Y, Souza JP, Stegemann M, Ugarte S, Venkatapuram S, Vuyiseka D, Preller J, BrignardelloPetersen R, Kum E, Qasim A, Zeraatkar D, Owen A, Guyatt G, Lytvyn L, Diaz J, Vandvik PO, Jacobs M (2021) A living WHO guideline on drugs to prevent covid-19. 372:n526. BMJ (Clinical research ed)

36. Chalmers JD, Crichton ML, Goeminne PC, Cao B, Humbert M, Shteinberg M, Antoniou KM, Ulrik CS, Parks H, Wang C, Vandendriessche T, Qu J, Stolz D, Brightling C, Welte T, Aliberti S, Simonds AK, Tonia T, Roche N (2021) Management of hospitalised adults with coronavirus disease 2019 (COVID19): a European Respiratory Society living guideline. The European respiratory journal 57

37. Tomazini BM, Maia IS, Cavalcanti AB, Berwanger O, Rosa RG, Veiga VC, Avezum A, Lopes RD, Bueno FR, Silva M, Baldassare FP, Costa ELV, Moura RAB, Honorato MO, Costa AN, Damiani LP, Lisboa T, 
Kawano-Dourado L, Zampieri FG, Olivato GB, Righy C, Amendola CP, Roepke RML, Freitas DHM, Forte DN, Freitas FGR, Fernandes CCF, Melro LMG, Junior GFS, Morais DC, Zung S, Machado FR, Azevedo LCP (2020) Effect of Dexamethasone on Days Alive and Ventilator-Free in Patients With Moderate or Severe Acute Respiratory Distress Syndrome and COVID-19: The CoDEX Randomized Clinical Trial. JAMA 324:1307-1316

\section{Tables}

Due to technical limitations, Table 1 is only available as a download in the Supplemental Files section.

\section{Figures}

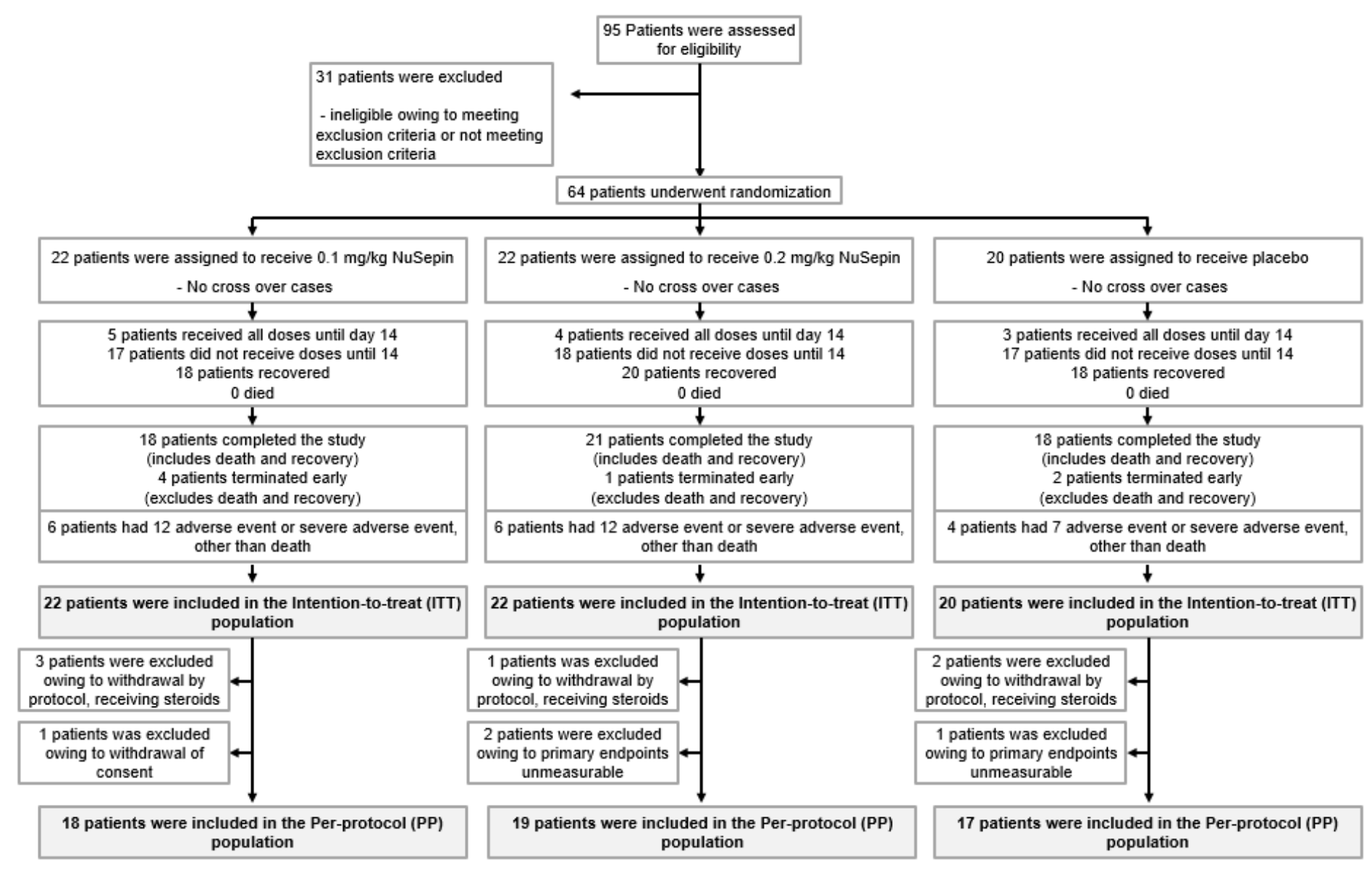

\section{Figure 1}

Legend not included with this version. 
RR $\left[95 \% \mathrm{Cl}^{1}{ }^{1} \mathrm{p}\right.$ value $^{2}$

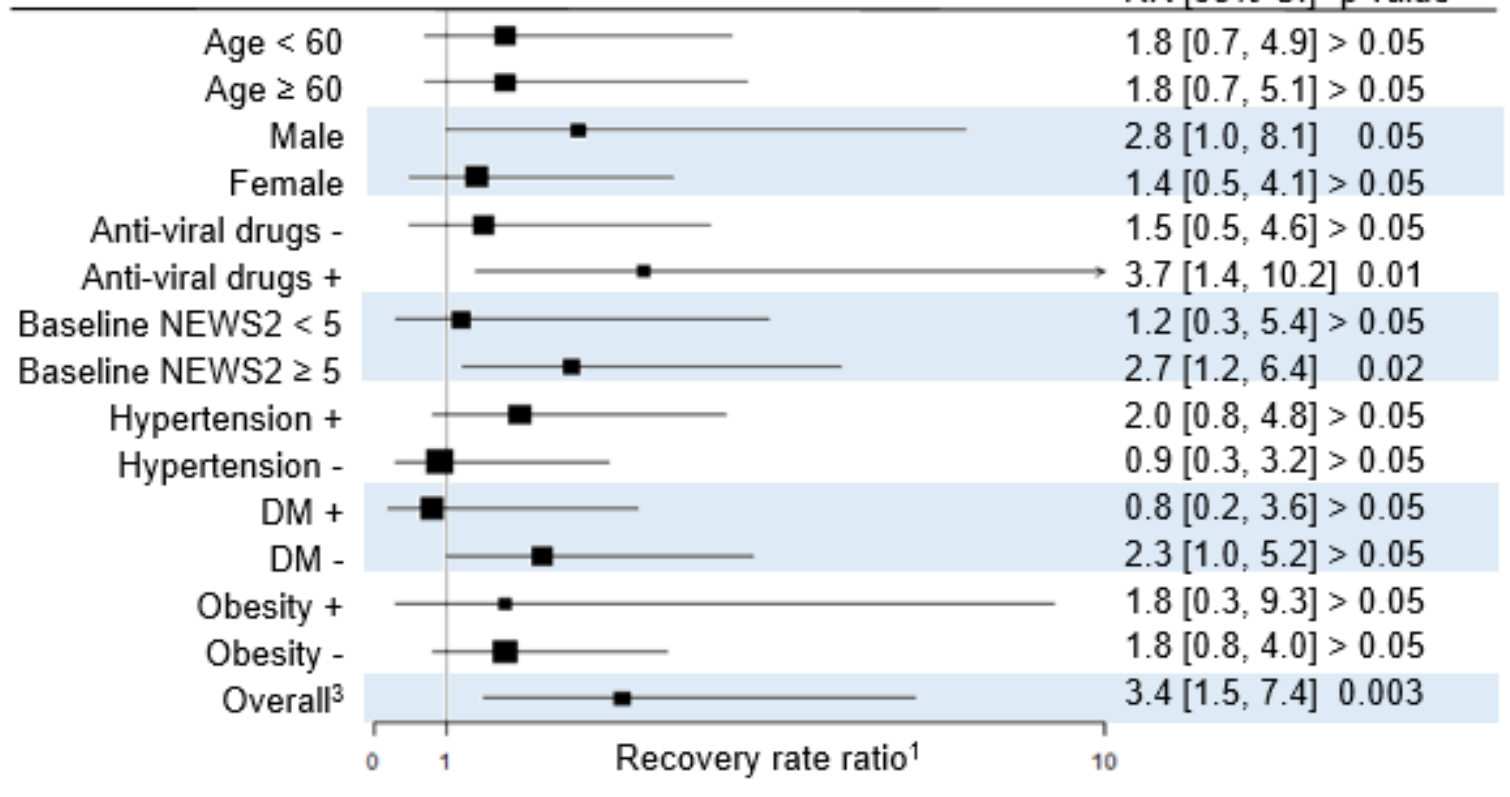

Figure 2

1Recovery rate ratios [95\% C.I.] between placebo and $0.2 \mathrm{mg} / \mathrm{kg}$ NuSepin group in PP set were compared. The clinical recovery was defined by TTCI_S, time to complete clinical remission when NEWS2 $=0$ maintained for $24 \mathrm{hrs}$. Recovery rate ratios (=hazard ratio) and $2 p$ values were determined by Cox proportional hazards model. A Recovery rate ratios $>1$ favors the NuSepin group in recovery rate compared with placebo group. 3Recovery rate ratio of all patients in a PP set were analyzed after adjusting effects of covariates [anti-viral drugs and disease severity (base line NEWS2 $\geq 5$ )] by using Cox regression analysis. 
A

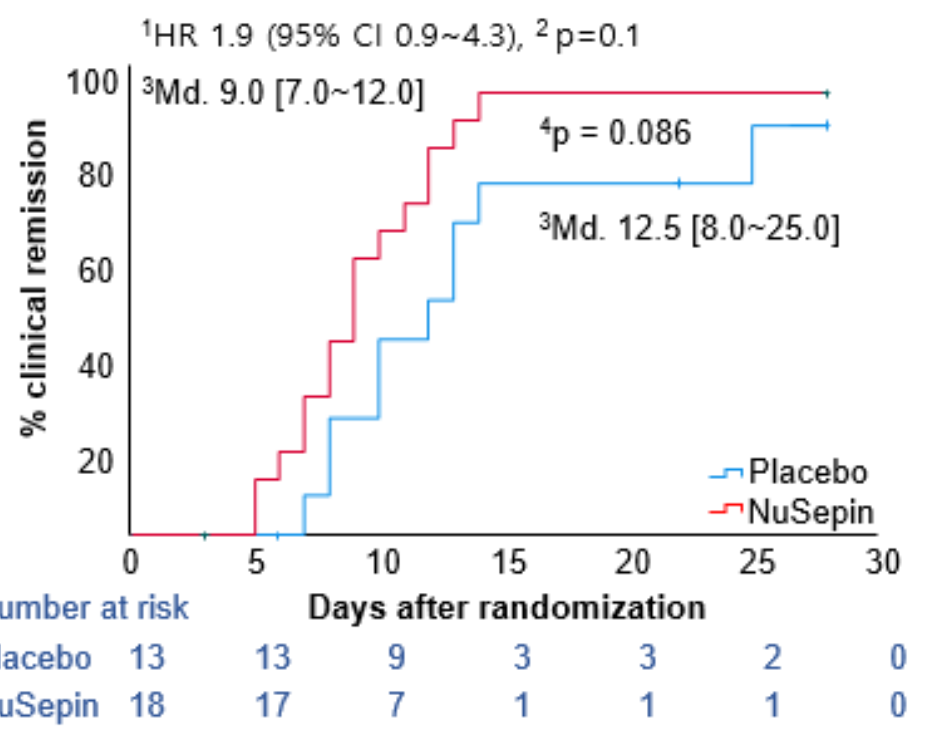

B

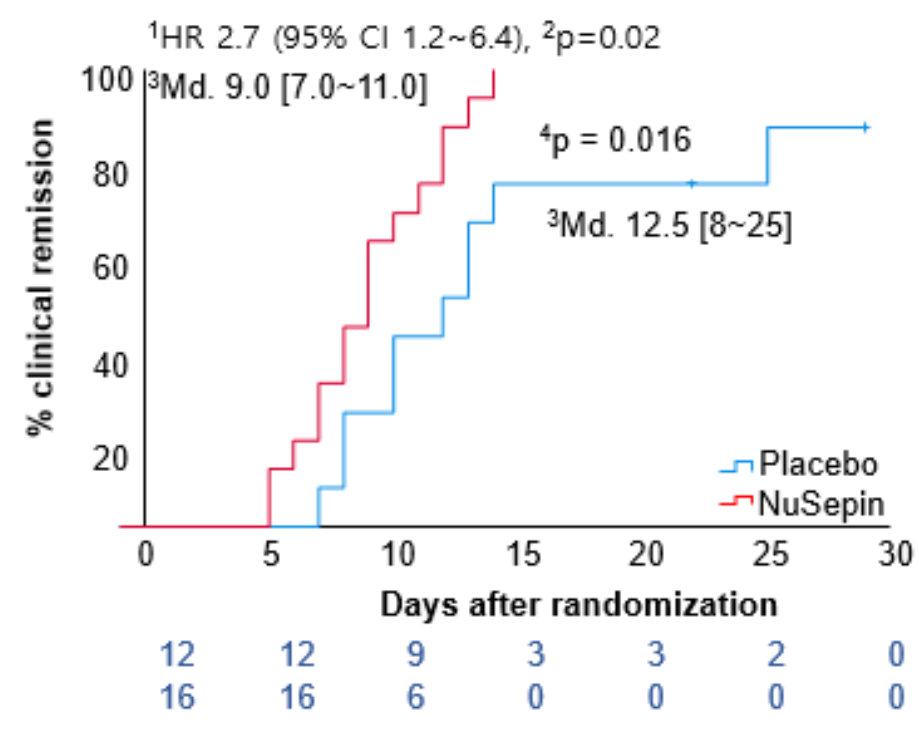

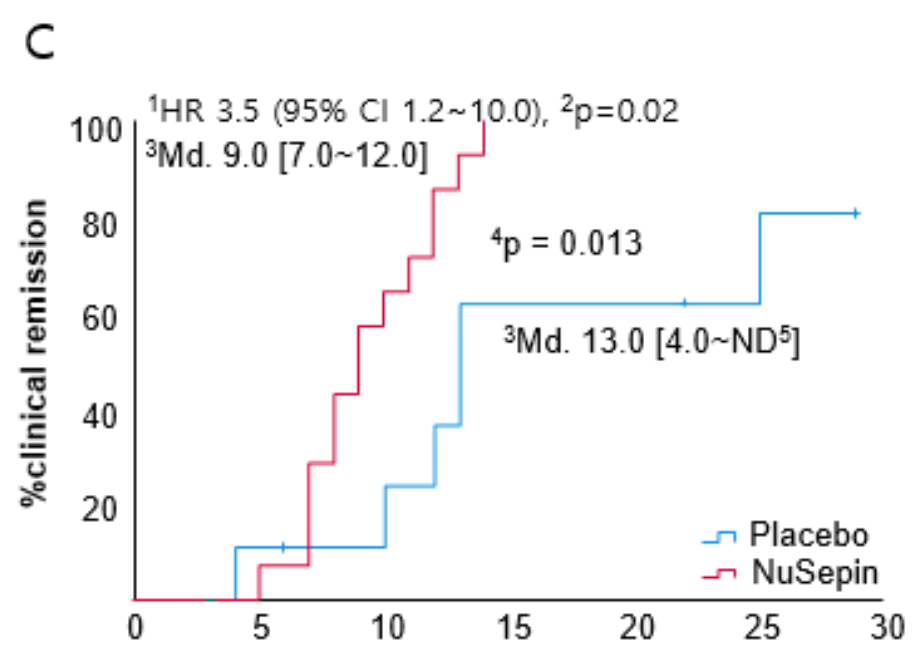

Number at risk

$\begin{array}{cccccccc}\text { Placebo } & 9 & 8 & 7 & 3 & 3 & 2 & 0 \\ \text { NuSepin } 14 & 14 & 6 & 0 & 0 & 0 & 0\end{array}$

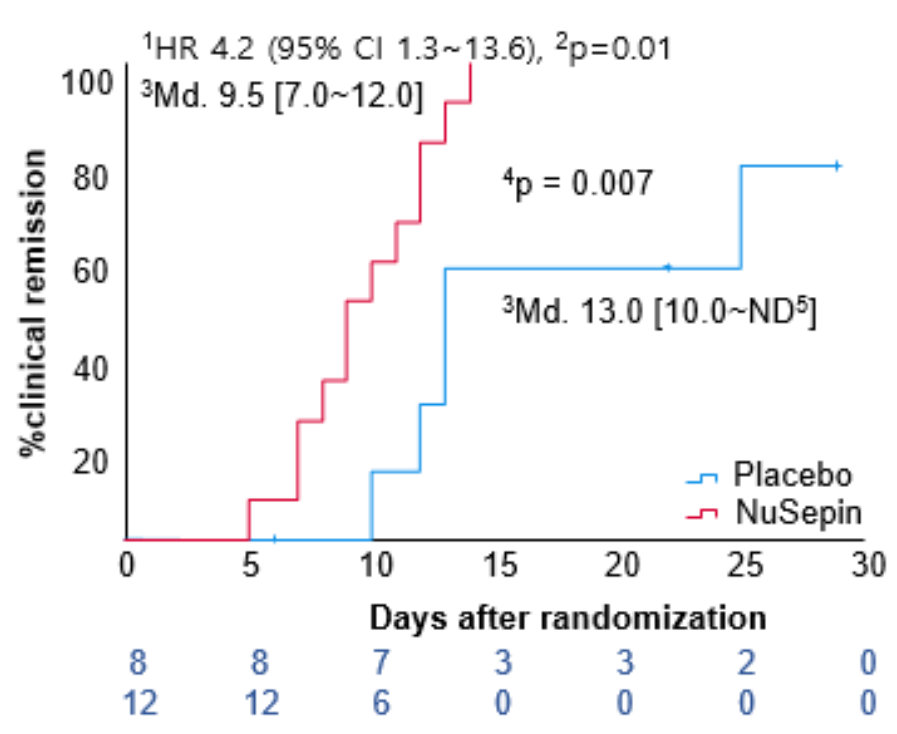

\section{Figure 3}

$\%$ clinical remission of patients in two cohorts, placebo and $0.2 \mathrm{mg} / \mathrm{kg}$ NuSepin. A. The patients in ITT population with baseline NEWS $2 \geq 5$ were analyzed by Kaplan-Meier survival analysis. B. The patients in PP population with baseline NEWS $2 \geq 5$ were analyzed by Kaplan-Meier survival analysis. The clinical remission is defined by NEWS2 $=0$ maintained for $24 \mathrm{hrs}$. C. The patients in ITT population who received anti-viral drugs were analyzed. D. The patients with baseline NEWS $2 \geq 5$ and who received anti-viral drugs in ITT population were analyzed. The clinical remission is defined by NEWS2 $=0$ maintained for $24 \mathrm{hrs}$. Antiviral drugs are Lopinavir-Ritonavir or Favipiravir. The patients who received two or more antiviral drugs are not included. 1 Hazard ratio and $2 p$ values were determined by Cox proportional hazards 
model. A hazard ratio $>1$ favors the NuSepin group in recovery rate. 3median [95\% C.I.], and $4 p$ values are from Log-Rank test (Mantel-Cox) in Kaplan-Meier survival analysis. 5ND, not determined due to outlier.
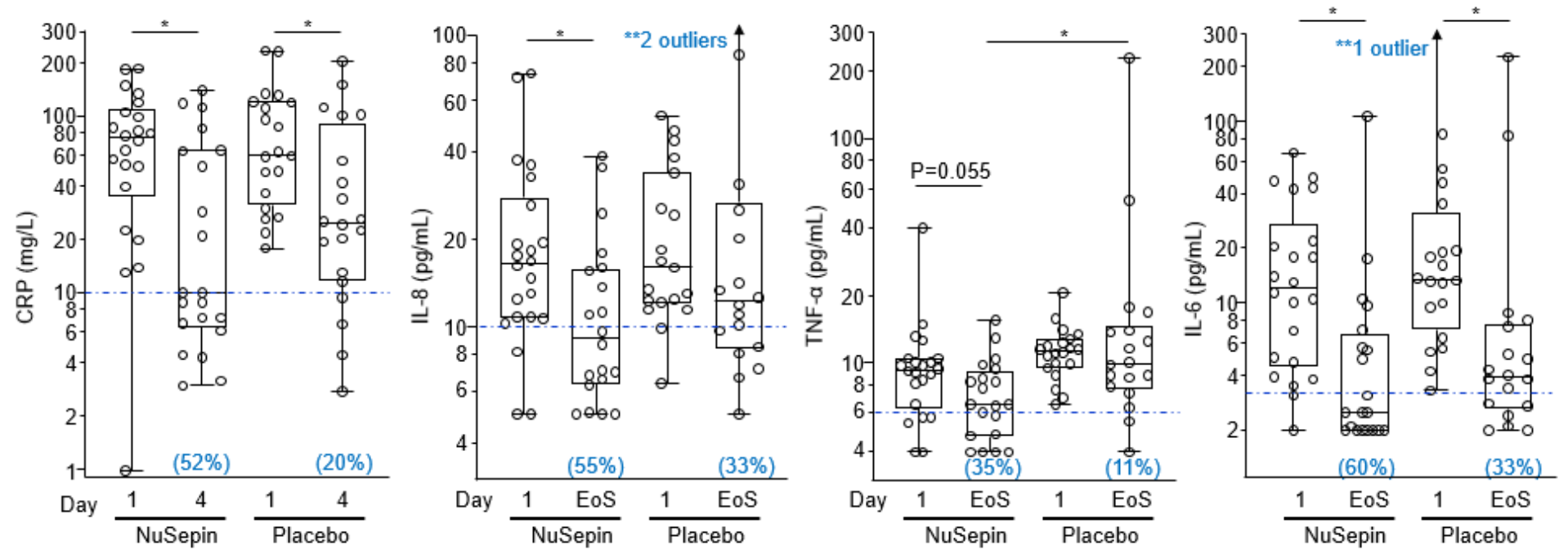

\section{Figure 4}

Blood levels in two cohorts of patients, placebo and $0.2 \mathrm{mg} / \mathrm{kg}$ NuSepin, at day 4 or day of end of study (EoS) compared with those at randomization day (day 1). All patients in ITT population were analyzed. \% in parenthesis denote $\%$ of patients with normal blood levels (CRP $<10 \mathrm{mg} / \mathrm{ml}$, IL-8 $<10 \mathrm{pg} / \mathrm{ml}$, TNF-a $<6$ $\mathrm{pg} / \mathrm{ml}$, and IL- $6<3.3 \mathrm{pg} / \mathrm{ml}$ ) at day 4 or at the day of EoS; the boxes represent median and interquartile range; the whiskers extend to the highest and lowest values within $1.5 \mathrm{x}$ the interquartile range of the nearer quartile; and the dots represent observed values. ${ }^{*} \mathrm{p}<0.05$ by Wilcoxon Rank sum test. ${ }^{*}$ Arrows at the end of error bars denote presence of outliers, defined by $>3 x$ standard error of the mean.

\section{Supplementary Files}

This is a list of supplementary files associated with this preprint. Click to download.

- Table1.png

- SupplementaryfigureTables.pptx 